of quality of life in asthma are also of a similar order. ${ }^{11}$ Such changes are therefore likely to be relevant to patients but cannot be ascribed to airway bronchodilation since $\mathrm{FEV}_{1}$ was unchanged. This then begs the questions as to what is being changed that makes patients feel better? Indeed, what was the abnormality that made these patients symptomatic in the first place? Is it part of the asthma or is it a concurrent problem?

Several discrete aspects need to be examined. The science around breathing control in the ambulant state remains quite limited. Chronic hyperventilation states are recognised, but the balance between psychic and somatic contributions to the overall state remains unclear. $^{12}{ }^{13}$ Some work does point towards respiratory physiological abnormalities, ${ }^{14}$ but there are few definitive answers. Next there is the role of breathing control and asthma. Asthma is hard to define and is multifactorial. Separating potential breathing control abnormalities from the airway effects of allergy, irritants, inflammation and exercise is challenging. And the questions continue as to whether it was the full Papworth technique or one component of it that produced the change.

However, regardless of whether the answers lie in a new physiological explanation or in an understanding of psychological reactions to the presence of a disease, the finding that something has made patients feel better means we cannot ignore this challenge. Five hours of therapist time for benefits that are apparent at 12 months compares well with the cost of a long-acting $\beta$ agonist for 1 year.

But these are difficult studies to deliver; adequate placebo control is difficult to contrive and they are time consuming to complete. It is an area that has little appeal for pharmaceutical or commercial support and it is hard to set out research protocols that will appeal to grant givers. Without financial support, the hurdles imposed by research governance, ethics committees and NHS pressures to work to targets mean that further studies may not be forthcoming. These workers are to be congratulated on completing this study without financial support.

Holloway and West's paper is not definitive and is insufficient to change management recommendations on its own. But the evidence that symptoms are not fully controlled by pharmacotherapy and the fact that many patients vote with their feet (and wallets) to seek outand apparently benefit from-alternative therapies should stimulate more studies. Maybe the time has come to tackle these symptoms from a different angle and to understand how psychology and the forgotten science of physiology may be as important to people with asthma as is the understanding of allergy and inflammation.

\section{Thorax 2007;62:1033-1034.}

doi: $10.1136 /$ thx.2007.084707

Correspondence to: Professor Mike G Pearson, Clinical Sciences Centre, University Hospital

Aintree, Longmoor Lane, Liverpool L9 7AL, UK; michael.pearson@liverpool.ac.uk
Competing interests: None.

\section{REFERENCES}

1 Holloway EA, West RJ. Integrated breathing and relaxation training (the Papworth method) for adults with asthma in primary care: a randomised controlled trial. Thorax 2007;62:1039-42.

2 Folgering $\mathbf{H}$. The hyperventilation syndrome. In: Altose MD, Kawakami Y, eds. Control of breathing in health and disease. New York, Basel: Marcel Dekker, 1999:633-60.

3 Howell JBL. Behavioural breathlessness. Thorax 1990:45:287-92.

4 Lum LC. The syndrome of habitual chronic hyperventilation. Recent Adv Psychosom Med 1976;3:196-230.

5 Sterk PJ, Fabbri LM, Quanjer PJ, et al. Airway responsiveness: standardised challenge testing with pharmacological, physicial and sensitising stimuli in adults. Eur Respir J, 1994:6(Suppl 16), 53-83.

6 European Community Respiratory Health Survey. Variations in the prevalence of respiratory symptoms, self-reported asthma attacks, and use of asthma medication in the European Community Respiratory Health Survey (ECRHS). Eur Respir J 1996;9:687-95.

7 Demeter SL, Lyons HA. Hyperventilation syndromes and asthma. Am J Med 1986;81:989-94.

8 Ernst E. Complementary therapies for asthma: what patients use. J Asthma 1998;35:667-71.

9 Holloway E, Ram F. Breathing exercises for asthma. In: The Cochrane Library, Issue 1. Oxford: Update Publications, 2004.

10 Jones PW, Bosch TK Quality of life changes in COPD patients treated with salmeterol. Am J Respir Crit Care Med 1997; 155:1283-9.

11 Rutten-van Molken MP, Custers F, van Doorslaer EK, et al. Comparison of performance of four instruments in evaluating the effects of salmeterol on asthma quality of life. Eur Respir J 1995;8:888-98.

12 Garder W. Orthostatic increase of respiratory gas volumes in hyperventilation syndromes. Thorax 2000;55:257-9.

13 Thomas M. Hyperventilation syndrome. Thorax 2005; $55: 884$

14 Jack S, Rossiter HB, Pearson MG, et al. Ventilatory responses to inhaled carbon dioxide, hypoxia and exercise in subjects with idiopathic hyperventilation Am J Respir Crit Care Med 2004;170:1 18-25.

\title{
What do non-eosinophilic asthma and airway remodelling tell us about persistent asthma?
}

\section{Peter G Gibson}

\section{There is still much more to learn about the pathogenesis and treatment of asthma}

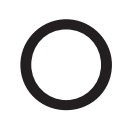
ver a decade of careful clinicopathological investigation has characterised the allergen-triggered Th2 response in asthma that leads to eosinophilic airway inflammation. This research has directed drug discovery programmes and we now have effective treatment for most steps in the eosinophilic asthma pathway. This list includes interventions that act at discrete levels such as allergen avoidance, allergen immunotherapy, anti-IgE antibodies, anti-interleukin-5 monoclonal antibodies and leucotriene receptor antagonists, together with corticosteroids that act on multiple levels in the pathway. Despite this significant success in therapeutic discovery, asthma persists. There must be something more to the pathogenesis of asthma. What could it be?

Airway remodelling and non-eosinophilic asthma (NEA) are both topical answers to this question. To date these have been pursued as distinct entities, but the paper by Berry and colleagues ${ }^{1}$ published in this issue of Thorax (see $\boldsymbol{p}$ 1043) addresses both issues and allows consideration of the interaction and overlap between airway remodelling and inflammatory subtype in asthma.

NEA refers to an asthma subtype where patients exhibit asthma symptoms and abnormal airway physiology (airway hyperresponsiveness (AHR), variable airflow obstruction) in the absence of a significant airway eosinophilia. ${ }^{2}$ Its importance arises because NEA is common, it seems to have a different 
pathogenesis from allergen-induced asthma $^{3}$ and it may be relatively resistant to corticosteroid therapy. ${ }^{4}$

Airway remodelling in asthma refers to changes in structural components of the airway wall and is believed to result in fixed airflow obstruction, persistent AHR and a poor short-term treatment response. Typical features are fibrosis of the subepithelial basement membrane, mucus gland hyperplasia and changes in airway smooth muscle.

Berry et al investigated the relationship between features of airway remodelling in asthma, where subjects were classified by their underlying inflammatory subtype. When viewed in the context of other studies in chronic cough ${ }^{5}$ and refractory asthma, ${ }^{6}$ a consistent pattern begins to emerge.

First, the concept that the eosinophil pathway causes AHR now seems untenable. Several studies clearly show that AHR can occur in the absence of eosinophilic infiltration of the airway mucosa or the airway lumen, ${ }^{16}$ and that eosinophilic bronchitis occurs without accompanying AHR. ${ }^{5}$ What seems more plausible is that eosinophilic inflammation can modulate the degree of airway responsiveness along a continuum, explaining how steroid treatment and allergen exposure can shift airway responsiveness both within and outside the asthmatic range. ${ }^{7}$ So what else determines AHR? Remodelled airway smooth muscle looms large here, and the observations of Berry et al ${ }^{1}$ both confirm and extend recent observations that a mast cell infiltrate into airway smooth muscle is associated with AHR. ${ }^{5}$ That this is the case in eosinophilic asthma is expected, but what Berry et al show is that, in NEA where eosinophils are absent from both the airway lumen and the airway mucosa, the presence of AHR is accompanied by a mast cell infiltration of the airway smooth muscle.

Second, the concept that a hallmark feature of asthma is increased thickness of the fibrous layer beneath the epithelial basement membrane (subepithelial fibrosis) is also untenable. This feature is not restricted to asthma since it occurs in airway diseases associated with eosinophilic inflammation but not asthma such as allergic rhinitis and cough with eosinophilic bronchitis. In subjects with NEA, the thickness of the subepithelial fibrous tissue was the same as in normal subjects, and the only patients in whom it was increased were those with eosinophilic asthma. Thus, subepithelial fibrosis may be the result of eosinophilic bronchitis, whatever the accompanying clinical phenotype. This is biologically plausible given evidence from animal models and known profibrotic cytokines released by eosinophils. ${ }^{8}$ That AHR with mast cell myositis is distinct from subepithelial fibrosis indicates a separate regulatory pathway for these two features of airway wall remodelling and suggests that different treatments will be needed for these separate components of airway disease.

Third, the concept that NEA is poorly responsive to corticosteroid treatment is strengthened by the small randomised controlled trial conducted by Berry et al where the response to inhaled corticosteroid was examined with stratification by inflammatory phenotype. The results confirm those of others that eosinophilic asthma is a highly steroid-responsive condition. Importantly, the study provides the first evidence from a randomised controlled trial that NEA is poorly responsive to short-term administration of inhaled corticosteroid. This has important practical implications for the treatment of people with asthma, and provides a pathophysiological explanation for the beneficial effects seen when asthma is managed using sputum cell counts to adjust treatment. ${ }^{910}$ This may also be a useful explanation for the synergy seen when inhaled corticosteroids are combined with long-acting $\beta$ agonists in asthma. Given the high prevalence of NEA, the combination of inhaled corticosteroids and long-acting $\beta$ agonists would provide effective treatment for both phenotypes where inhaled steroids treat eosinophilic asthma and long-acting $\beta$ agonists control the AHR seen in NEA, as well as partially suppressing interleukin-8-mediated neutrophilic inflammation. ${ }^{11}$

The relative steroid resistance of NEA is still, however, a contentious issue. Uncontrolled studies disagree on whether there is a difference in clinical outcomes after steroid treatment between eosinophilic asthma and NEA. The lack of an effect in uncontrolled studies could be explained by several design flaws that are addressed by using a randomised controlled trial. A larger randomised controlled trial would strengthen the conclusion that NEA is relatively unresponsive to inhaled corticosteroid.

\section{HOW SHOULD NEA BE RECOGNISED?}

This is an important question, since the subtype represents the occurrence of asthma symptoms and abnormal physiology in the absence of eosinophilic inflammation. Several variables could confound the diagnosis of NEA, and these relate to causes of transient neutrophilic bronchitis and other diseases associated with neutrophilic bronchitis which include viral infection, bronchiectasis and potentially chronic obstructive pulmonary disease. While these factors will need to be considered in research studies, they may be of lesser importance in clinical practice where the purpose of "inflammometry" is to guide treatment.

Certain asthma triggers, such as rhinovirus infection, can result in a transient neutrophilic bronchitis. This can suppress a sputum eosinophilia, and the sputum eosinophilia returns once the infection has resolved. ${ }^{12}{ }^{13}$ Classification of the phenotype during the infection may be erroneous, so the diagnosis of NEA should be made with preferably more than one assessment. Just how many assessments are needed to identify a stable inflammatory subtype requires more work, but will be of immense practical importance. The role of mixed phenotypes (eosinophil and neutrophil) also warrants more attention. These are uncommon in stable asthma but may be more important in complicated asthma. ${ }^{2}$

\section{WHAT IS THE BEST ASSAY TO RECOGNISE NEA?}

At present this seems to be induced sputum. While Berry et al show that biopsy or bronchoalveolar lavage could also be used, a similar study by Lemiere et $a l^{14}$ found sputum to be more reliable. The ideal situation would be a marker that can be used to positively identify the eosinophilic and non-eosinophilic subtypes. The fraction of expired nitric oxide $\left(\mathrm{FE}_{\mathrm{NO}}\right)$ may be useful in steroid-naïve individuals since $\mathrm{FE}_{\mathrm{NO}}$ levels were normal in NEA and raised in eosinophilic asthma. However, the usefulness of $\mathrm{FE}_{\mathrm{NO}}$ in subtype classification in steroid-treated asthma remains to be determined. The positive identification of NEA requires a marker that is positive in NEA and negative in eosinophilic asthma. Some promising candidates are neutrophil elastase and interleukin- 8 which are increased in the neutrophil subtype of NEA. ${ }^{15}$

In conclusion, the persistence of asthma teaches us that there is much more to learn about the pathogenesis and treatment of this much studied condition. Studying inflammatory subtypes is a useful approach to this problem that is paying dividends both in understanding the mechanisms of persistent asthma and relating these to clinical management.

Thorax 2007;62:1034-1036.

doi: 10.1136/thx.2007.079061

Correspondence to: Professor Peter G Gibson, Level 3, Hunter Medical Research Institute, John Hunter Hospital, Locked Bag 1, Hunter Region Mail Centre, NSW 2310 Australia; peter. gibson@hnehealth.nsw.gov.au

Competing interests: None. 


\section{REFERENCES}

1 Berry M, Morgan A, Shaw DE, et al. Pathological features and inhaled corticosteroid response of eosinophilic and non-eosinophilic asthma. Thorax 2007;62:1043-9.

2 Simpson JL, Scott R, Boyle MJ, et al. Inflammatory subtypes in asthma: assessment and identification using induced sputum. Respirology 2006;11:54-61.

3 Simpson JL, Grissell TV, Douwes J, et al. Innate immune activation in neutrophilic asthma and bronchiectasis. Thorax 2007;62:211-8.

4 Pavord ID, Brightling CE, Woltmann G, et al. Noneosinophilic corticosteroid unresponsive asthma. Lancet 1999;353:2213-4.

5 Brightling CE, Bradding P, Symon FA, et al. Mastcell infiltration of airway smooth muscle in asthma. N Engl J Med 2002;346:1699-705.
6 Wenzel SE, Schwartz LB, Langmack EL, et al. Evidence that severe asthma can be divided pathologically into two inflammatory subtypes with distinct physiologic and clinical characteristics. Am J Respir Crit Care Med 1999;160:1001-8.

7 Gibson PG. Airway hyperresponsiveness in asthma. Thorax 1999;54:656-7.

8 Phipps S, Benyahia F, Ou TT, et al. Acute allergen-induced airway remodeling in atopic asthma. Am J Respir Cell Mol Biol 2004;31:626-32.

9 Jayaram L, Pizzichini MM, Cook RJ, et al. Determining asthma treatment by monitoring sputum cell counts: effect on exacerbations. Eur Respir J 2006;27:483-94.

10 Green RH, Brightling CE, McKenna S, et al. Asthma exacerbations and sputum eosinophil counts: a randomised controlled trial. Lancet 2002;360:1715-21.
11 Maneechotesuwan K, Essilfie-Quaye S, Meah S, et al. Formoterol attenuates neutrophilic airway inflammation in asthma. Chest 2005;128:1936-42.

12 Grissell TV, Powell H, Shafren DR, et al. Interleukin 10 gene expression in acute virus-induced asthma. Am J Respir Crit Care Med 2005; 172:433-9.

13 Gauvreau GM, Inman MD, Kelly M, et al. Increased levels of airway neutrophils reduce the inhibitory effects of inhaled glucocorticosteroids on allergen-induced ainway eosinophils. Can Respir J 2002;9:26-32.

14 Lemiere C, Ernst P, Olivenstein R, et al. Airway inflammation assessed by invasive and noninvasive means in severe asthma: eosinophilic and noneosinophilic phenotypes. J Allergy Clin Immunol 2006;1 18:1033-9.

15 Simpson JL, Scott RJ, Boyle MJ, et al. Differential proteolytic enzyme activity in eosinophilic and neutrophilic asthma. Am J Respir Crit Care Med $2005 ; 172: 559-65$

\section{Cannabis and the lung}

\section{Peter Lange}

\section{Cannabis smoking constitutes a substantial hazard to the lung}

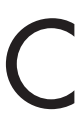
annabis (or marijuana) is not only the most widely used illegal drug in the western world but, after tobacco, also the most commonly smoked substance. In the UK almost 50\% of young adults have tried to smoke cannabis at some time. ${ }^{1}$ Among people aged 1630 years of age there is a substantial number of frequent users, in some populations in the range of about 5\%. The active substance responsible for the psychostimulating effect of cannabis is delta ${ }^{9}$-tetrahydrocannabinol

(THC). However, as with tobacco smoke, cannabis smoke consists of a large mixture of compounds including polycyclic aromatic hydrocarbons, carbon monoxide, cyanide, benzene and many others.

Cannabis is prepared from the hemp plant which-especially in the 19th and the beginning of the 20th century-was grown for industrial purposes in order to produce fibres, but it has gradually been replaced by other coarse-fibre plants. Archaeological findings show that cannabis was used in many ancient cultures in spiritual and religious contexts as a psychostimulating and trance-inducing drug. Later, in the 19th century, it was promoted for its medical properties including pain-relieving, antiemetic and anticonvulsant effects. Yet, after the invention of aspirin and other more effective drugs, the use of cannabis as a popular medical drug declined.

The so-called recreational use of cannabis became more widespread during the golden period of jazz in the 1920s and 1930s and later became a part of the youth culture in the 1960s. Although cannabis can be prepared for consumption in several forms (beverages, cakes, oils), the most usual intake is by inhalation through the lungs. Cannabis can be smoked in cigarettes (joints), pipes or in special devices such as bong or chillum. Irrespective of the device, the technique of smoking cannabis differs from smoking regular tobacco with larger puffs, deeper inhalation and greater breath holding time, sometimes accompanied by valsalva manoeuvres to achieve a higher systemic absorption of THC. In fact, this smoking technique (rather than cannabis itself) has been proposed as the mechanism responsible for cases of spontaneous pneumothorax and bullous lung disease reported in young cannabis smokers. $^{2}$ Most importantly, however, this smoking technique results in a far greater deposition of toxic substances in the lung than with regular tobacco smoking. ${ }^{3}$

The number of studies on the pulmonary effects of cannabis is quite limited. In particular, in contrast to the worldwide research on tobacco, relatively few research groups have conducted relevant studies on the pulmonary effects of cannabis. Most of our knowledge comes from the University of Southern California where Tashkin et al ${ }^{4}$ have, since the early 1970s, performed several experimental, clinical and epidemiological studies. However, there is now an increasing focus on the possible harmful effects of cannabis on the lung. A recent systematic review of the literature identified 34 relevant publications evaluating either short-term or long-term effects of cannabis smoking on pulmonary function and respiratory symptoms. ${ }^{5}$ This review confirms that, although cannabis smoking results in an acute bronchodilation, it exerts very potent inflammatory effects on the airways which, in the longer term, result in a very high prevalence of cough, sputum and wheeze. These clinical symptoms are paralleled by bronchoscopic findings showing mucosal swelling and erythema, increased airway secretions, goblet cell hyperplasia, loss of ciliated epithelium, squamous metaplasia and an increased number of alveolar macrophages with impaired microbiocidal activity. ${ }^{4}$ The latter finding is consistent with case reports of opportunistic pulmonary infections in cannabis smokers.

With regard to the risk of developing respiratory cancer, the evidence is more controversial. Yet, as cannabis smoke contains similar carcinogens to tobacco smoke and the smoking technique results in an even higher concentration and the deposition in the airways of inhaled particles, it is likely that cannabis smoking could cause airway malignancies. However, a large epidemiological study failed to show an increased risk in cannabis smokers, but this study has been criticised by others because the follow-up period was too short. ${ }^{4}$

The findings regarding the long-term effects of cannabis smoking on pulmonary function are also conflicting and previous reviews have concluded that data on an association between cannabis smoking and reduced pulmonary function are inconclusive. ${ }^{45}$ In this issue of Thorax (see $p$ 1058), Aldington et $a l^{6}$ present new data on this important problem. They compared lung function and high-resolution CT (HRCT) scans of 